\title{
COMMUNICATION
}

\section{New Directions in \\ Numerical Computation}

\section{Tobin A. Driscoll, Endre Süli, and Alex Townsend, Editors}

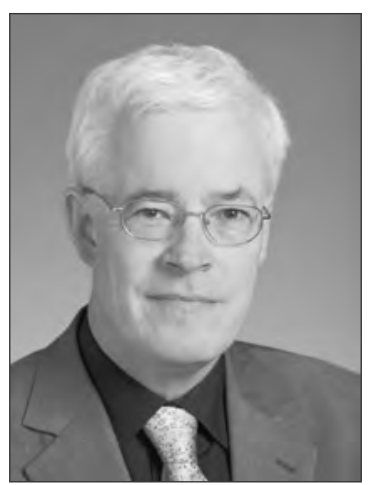

Nick Trefethen
In August 2015 a distinguished collection of numerical analysts gathered at Oxford to celebrate the sixtieth birthday of Nick Trefethen FRS and consider the future of numerical analysis. Some of the plenary speakers provided short essays for Notices. The full collection is online. ${ }^{1}$

In a 1992 essay, "The Definition of Numerical Analysis", 2 Trefethen writes of the field, "[O]ur central mission is to compute quantities that are typically uncomputable, from an analytical point of view, and to do it at lightning speed." These essays explore a few of the particulars of that mission.

\section{Jean-Paul Berrut}

\section{A Puzzling Question about Numerical Analysis}

Why are so many academic mathematicians content with piecewise smooth approximations to solutions to functional equations when those solutions are known a priori to be smooth? Chebfun impressively and beautifully demonstrates the effectiveness of smooth one-dimensional approximants. These may oscillate more than splines of comparable accuracy, but their convergence is faster and automatically adjusts to the smoothness of the underlying function. In contrast, the

Tobin A. Driscoll is professor of mathematical sciences at the University of Delaware. His email address is drisco11@ude1.edu.

1 tobydrisco11.net/newdirections2015.
${ }^{2}$ LN Trefethen, "The Definition of Numerical Analysis"
SIAM News, November 1992.

For permission to reprint this article, please contact: reprint-permission@ams.org.

DOI: http://dx.doi.org/10.1090/noti1363 use of smooth functions in two and higher dimensions was, until recently, limited to relatively simple domains. Fundamental research on infinitely smooth two-dimensional interpolants may lead to interesting new approaches, yet the number of scientists working on them appears to be surprisingly small.

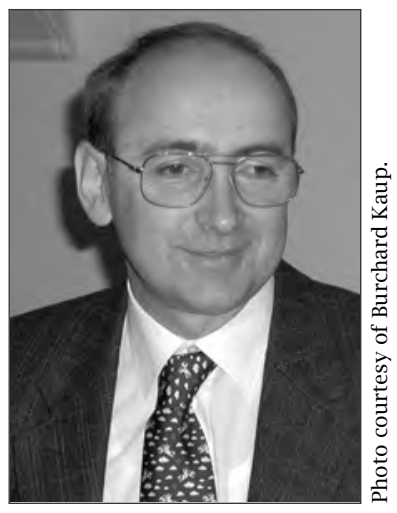

Jean-Paul Berrut

\section{Bengt Fornberg}

\section{The Method-Not the Machine}

It used to be said that improvements in scientific computing capabilities originate about equally from advances in algorithms and in hardware. During the last decade or two, the focus has shifted to building heroic-scale supercomputing facilities. Maybe this is partly because processing characteristics are quantifiable and easy to show in lists, with national and world records falling incessantly. However, the largest systems require inordinate amounts of power and infrastructure, and they also become obsolete very quickly. Yet algorithmic opportunities are as expansive as ever.

Endre Süli is professor of numerical analysis at the University of Oxford. His email address is su1i@maths.ox. ac.uk.

Alex Townsend is assistant professor of mathematics at Cornell University. His email address is ajt@mi t. edu .

Jean-Paul Berrut is professor of mathematics at the Université de Fribourg. His email address is jean-pau1. berrut@unifr.ch.

Bengt Fornberg is professor of applied mathematics at the University of Colorado. His email address is fornberg@colorado.edu. 


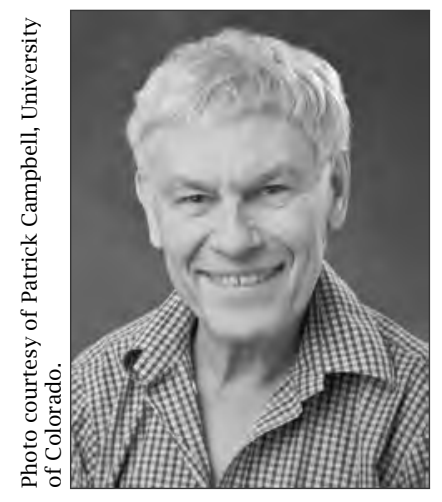

Bengt Fornberg
Private industry perpetuates legacy algorithms to a surprising extent despite the pressure of economic incentives. One example can be found in seismic exploration for oil and gas. Finite differences on regular grids for modeling wave propagation were updated from second to fourth order in the 1980s. Now twentieth-order finite differences are widely used for exploration production work, but material interfaces are still mostly treated only to first order. As a result, computations rely on extreme refinement to keep errors at a tolerable level. This approach has been fine-tuned on massive systems while largely ignoring the vast opportunities to exploit algorithmic improvements. Much the same holds for geoscientific simulations of the earth's systems, such as climate and weather.

What matters in the long run is: the Method-not the Machine.

\section{Anne Greenbaum}

\section{Return to the Days of Ptolemy}

In the coming years we will learn more about what can and cannot be predicted through analysis of

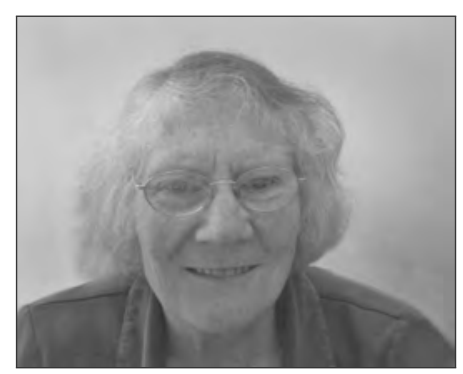

data, without necessarily formulating a realistic scientific model. Ptolemy was able to accurately predict planetary motions in this way. There is great interest today in predictions Anne Greenbaum of the stock market, the weather, our purchasing preferences, and so on, mainly or entirely through the analysis of data. This is good news for the numerical linear algebra community, as these computations require algorithms such as the singular value decomposition and rely on theoretical analysis connected with, for example, matrix completion problems.

It will be fascinating to see what new things we learn about ourselves. Will there come a day when I trust Amazon's book recommendations over my own instincts? What will be the effects

Anne Greenbaum is professor of applied mathematics at the University of Washington. Her email address is greenbau@amath.washington.edu. when gadgets that we do not understand start doing things that we do not expect?

This is not to say that data analysis will be the only means of understanding. As soon as predictability is established, scientists will be looking for explanations in terms of models and equations. This suggests a reversal of the prevailing order of inquiry, one that is already seen in mathematics. Conjectures are often formed based on computational experiments, and possible steps in the proof are tested numerically before too much time is spent trying to prove them.

\section{Nicholas J. Higham}

\section{Mixed Precision Computations}

For the last thirty years, most floating-point calculations in scientific computing have been carried out in IEEE double-precision arithmetic, which provides the elementary operations of addition, subtraction, multiplication, and division at a relative accuracy of about $10^{-16}$. We are now seeing growing use of mixed precision, in which different floating point precisions are combined in order to deliver a result of the required Nicholas J. Higham accuracy at minimal cost.

Single-precision arithmetic halves the costs of storing and transferring data and can take half the time of double-precision on the right hardware. Quadruple-precision arithmetic is supported by some compilers, and arbitrary-precision arithmetic is available in Fortran and $\mathrm{C}$ as well as Python, Julia, and MATLAB ${ }^{\mathrm{TM}}$.

Multiple-precision is being used for iterative refinement in linear algebra, rectification of algorithmic instability, and checking of results. We can expect these practices to expand and new ones to emerge as access to mixed precision becomes ever easier.

\section{Randy LeVeque}

\section{Sharing the Code}

A positive development in numerical analysis and scientific computing is the increasing interest in archiving and sharing computer programs that are

Nicholas J. Higham is Richardson Professor of Applied Mathematics at The University of Manchester. His email address is nick. higham@manchester.ac.uk.

Randy LeVeque is professor of applied mathematics at the University of Washington. His email address is $\mathrm{rj} 1$ @amath . washington. edu. 


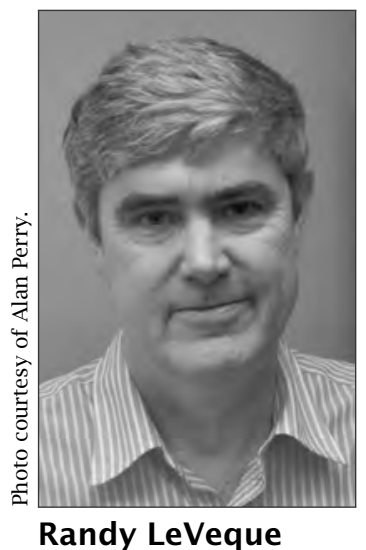

an integral part of research publications. In mathematics it is unthinkable to publish a theorem without including a carefully written proof. Unfortunately we have not had the same expectation for code written to test algorithms.

The "reproducible research" movement is attempting to address this. Journals and funding agencies are starting to require reproducibility, which is easier and more fun to accomplish than in the past due to improvements in technology, including open-source repositories such as GitHub, virtualization and cloud computing platforms, and browser-based notebooks for exposition.

Some people have been practicing reproducibility for decades, and Nick Trefethen serves as a good example. He has always enjoyed polishing his code to make it accessible and informative in research papers and exposition as well as in his software packages, from SCPACK to Chebfun. To choose just one example from his oeuvre, try to imagine his Spectral Methods in Matlab without the MATLAB ${ }^{\text {TM }}$. It would still be a valuable book, but without the code to learn from and experiment with, it would have had far less impact. We should all take such pride and pleasure in sharing our code!

\section{Ian H. Sloan}

\section{High Dimensionality-A New Direction for Numerical Analysis}

High-dimensional problems will be an important part of numerical analysis in the future-perhaps

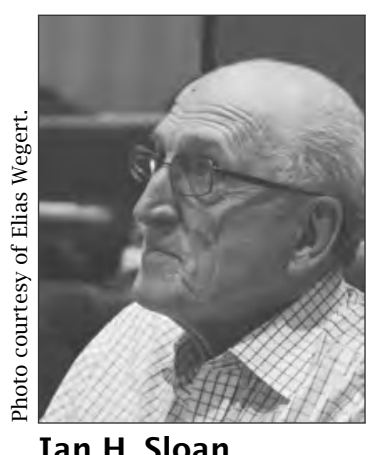

not a new one, because they were initiated by Norbert Wiener in 1938 . The numerical analysis of high dimensionality was further advanced in the 1950s and 1960s through the work of the number theorists Sobol, Hlawka, and Korobov, who (without any help from numerical analysts) initiated the subject of quasi-Monte Carlo integration in many dimensions. But high-dimensional problems have until now been a minor theme in numerical analysis. I think that this is destined to change.

A current major driver of change is uncertainty quantification. There are now journals devoted to this area. A good proportion of papers in these journals is currently on problems with moderate to

Ian H. Sloan is emeritus professor of mathematics at the University of New South Wales. His email address is I.S1oan@unsw.edu.au.

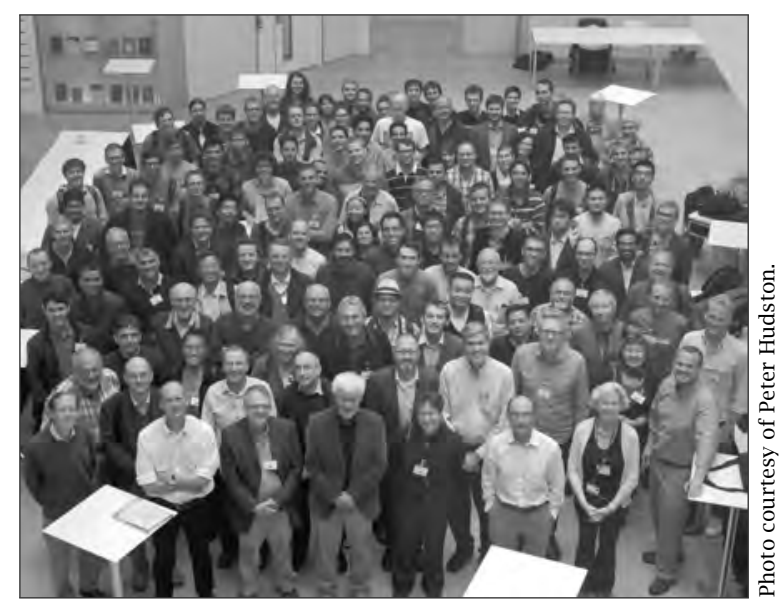

Attendees at the conference "New Directions in Numerical Computation," held at Oxford University in August 2015 in honor of Nick Trefethen's sixtieth birthday.

high dimensionality, with the prototype of Darcy flow through a porous medium. A random field, modeling for instance the permeability of an oil field, is naturally described by an infinite number of scalar random variables, giving rise to an infinitedimensional problem. A very large finite number of random variables may be needed to obtain a good approximation. Some buzzwords for the current crop of numerical methods are (generalized) polynomial chaos, stochastic Galerkin, stochastic collocation, sparse grids, multilevel Monte Carlo, quasi-Monte Carlo, and so on.

Why is all of this interest coming now? Because only now is the computing power available to tackle realistic problems. Why will interest increase in the future? Because such problems are inherently hard and suffer from the famous "curse of dimensionality". Now we are tackling only simple versions of such problems: for example, allowing only random fields with "finite-dimensional noise", small variance, and long correlation lengths. As computers become more powerful, some areas of numerical analysis may be effectively tamed, but high-dimensional problems never fully so. High-dimensional problems are here to stay. 